\title{
The Survival Benefit of Liver Transplantation
}

\author{
Robert M. Merion ${ }^{a, b}, *$, Douglas E. Schaubel ${ }^{b, c}$, \\ Dawn M. Dykstrab,d, Richard B. Freemane, \\ Friedrich K. Port ${ }^{b, d}$ and Robert A. Wolfe ${ }^{b, c}$ \\ a Department of Surgery, University of Michigan, Ann \\ Arbor, Michigan, USA \\ b Scientific Registry of Transplant Recipients, Ann Arbor, \\ Michigan, USA \\ ${ }^{c}$ Department of Biostatistics, University of Michigan, Ann \\ Arbor, Michigan, USA \\ 'University Renal Research and Education Association, \\ Ann Arbor, Michigan, USA \\ e Tufts-New England Medical Center, Boston, \\ Massachusetts, USA \\ * Corresponding author: Robert M. Merion, \\ merionb@umich.edu
}

Demand for liver transplantation continues to exceed donor organ supply. Comparing recipient survival to that of comparable candidates without a transplant can improve understanding of transplant survival benefit. Waiting list and post-transplant mortality was studied among a cohort of 12996 adult patients placed on the waiting list between 2001 and 2003. Timedependent Cox regression models were fitted to determine relative mortality rates for candidates and recipients. Overall, deceased donor transplant recipients had a $79 \%$ lower mortality risk than candidates (HR = $0.21 ; p<0.001)$. At Model for End-stage Liver Disease (MELD) 18-20, mortality risk was $38 \%$ lower (p $<0.01$ ) among recipients compared to candidates. Survival benefit increased with increasing MELD score; at the maximum score of 40 , recipient mortality risk was $96 \%$ lower than that for candidates $(p<0.001)$. In contrast, at lower MELD scores, recipient mortality risk during the first post-transplant year was much higher than for candidates (HR = 3.64 at MELD 6-11, HR = 2.35 at MELD 12-14; both $p<0.001$ ). Liver transplant survival benefit at 1 year is concentrated among patients at higher risk of pre-transplant death. Futile transplants among severely ill patients are not identified under current practice. With 1 year post-transplant follow-up, patients at lower risk of pre-transplant death do not have a demonstrable survival benefit from liver transplant.

Key words: Donor, liver transplantation, MELD, mortality, organs, SRTR, supply, survival benefit, waiting list

Received 25 June 2004, revised 6 September 2004 and accepted for publication 25 September 2004

\section{Introduction}

Liver transplantation has altered the natural history of endstage liver disease and is now considered the preferred therapy for a wide range of previously fatal chronic hepatic diseases. Optimal timing of liver transplantation in the course of disease is desirable to avoid harm from intervening too early and futility from transplanting too late. Unfortunately, little is known about the most appropriate time in the course of chronic liver disease to offer a transplant for the majority of patients, with the exception of primary biliary cirrhosis and other cholestatic disorders (1-3). Appropriate timing is especially important because of the uncertain availability of a donor organ engendered by the imbalance between the size of the pool of suitable recipients and the number of available deceased donors (4).

Given the severe shortage of donor organs, there is a need to identify which patients derive significant survival benefit from transplantation and which do not. As early as 1992, Delmonico et al. noted that assessment of outcome should be a component of allocation policy so that futile transplants could be avoided (5), and a recent report continued to support this view (6). To date, however, only pre-transplant mortality risk has been incorporated into liver allocation policy. The most recent change in liver allocation policy in the United States, by which candidates are ranked according to a more highly stratified index of pre-transplant mortality (7), continues to offer donor livers on this basis.

Comparisons of mortality risk among transplant recipients to mortality risk among listed transplant candidates with chronic end-stage renal disease, matching on time since initial listing, have been used to assess transplant survival benefit (8). This approach avoids inappropriate comparisons to the entire population of patients with end-stage disease, only a small fraction of whom are suitable candidates to be listed for transplantation. Registered transplant candidates who have already entered the transplant pathway constitute the best comparison group to transplant recipients.

In the field of renal transplantation, no distinctions are drawn among candidates based on medical urgency when allocation priority for deceased donor organs is being considered. This makes assessment of survival benefit more straightforward (8). In contrast, liver transplant allocation is based on the severity of the underlying disease necessitating hepatic replacement. Specifically, the current allocation 
scheme for candidates with chronic end-stage liver disease utilizes a measure of each patient's pre-transplant mortality risk that is calculated based on laboratory values of serum bilirubin, international normalized ratio of prothrombin time and serum creatinine. The score is derived from published data on the Model for End-stage Liver Disease (MELD), which predicts 3-month mortality risk for adult cirrhotics undergoing transjugular intra-hepatic portosystemic shunt procedures as well as other chronic liver disease populations and liver transplant candidates (9-11). A similar system (PELD) has been developed for pediatric candidates (12).

The tacit assumption that liver transplantation provides a large differential between waiting list mortality risk and post-transplant mortality risk for candidates at all MELD levels has not been previously tested at the national level. In this article, we use data from all liver transplant centers in the United States to examine the survival benefit attributable to liver transplantation, with a focus on adult patients with chronic liver disease at the low and high ends of the pre-transplant risk spectrum.

\section{Methods}

\section{Data sources}

This study used data from the Scientific Registry of Transplant Recipients (SRTR) regarding access to transplantation, waiting list mortality and posttransplant graft and patient survival, among all listed candidates and transplant recipients in the United States submitted by the members of the Organ Procurement and Transplantation Network (OPTN), and supplemented by mortality information from the Social Security Death Master File (13-15).

\section{MELD-based allocation system}

The national system for liver allocation operates according to a set of nested rules (16). Donor livers are first offered to candidates with acute liver failure or immediate post-transplant graft failure (Status 1). After Status 1, organs are allocated to candidates with chronic liver disease wait-listed at transplant centers within the donor's organ procurement organization (OPO) service area in descending MELD or PELD order, next to candidates in other OPOs in the region, and finally to candidates outside the region. MELD or PELD score data must be submitted periodically, with updates at more frequent intervals required at higher MELD or PELD scores (16). Regional review boards may consider requests for assigned (rather than calculated) MELD or PELD scores.

\section{Study population}

The study population included patients 18 years of age and older with an initial date of registration for deceased donor liver transplantation between September 2001 and June 2003 ( $n=12$ 996). The start of the study corresponded to the initial date of mandatory submission of the three components of the MELD score. Patients were followed to the earliest of death, loss to follow-up or the end of the observation period on June 30, 2003: post-transplant follow-up was a maximum of 1 year. Status 1 candidates were excluded $(n=626)$. Candidates with a diagnosis of hepatocellular carcinoma were excluded, since an arbitrary MELD score is assigned to such patients at listing. Patients who had MELD exception scores approved for use in lieu of calculated scores were censored at the date the exception score was granted $(n=1380)$. Since the study objective related to deceased donor transplantation, patient survival was also censored at the date of living donor liver transplant $(n=242)$.

Changes in calculated MELD scores for patients with chronic liver disease were tracked. A total of 67056 MELD scores was used for the analysis. Among the 12996 patients in the study, 12138 had more than one recorded MELD score.

\section{Analytic approach}

Patients contributed follow-up time to the candidate group before transplant and to the transplant group if and when they received a deceased donor liver transplant, and thereafter. Patients were censored at removal from the candidate pool, if removal was due to patient recovery $(n=120)$. Transplant recipients who experienced graft failure (with or without retransplantation) were not censored. Waiting list patients were classified based on their current MELD score. Waiting candidates contributed follow-up time at risk according to their most recent MELD score and could therefore accrue periods of follow-up time in multiple categories if their MELD score changed. Transplant recipients were classified based on their final pre-transplant MELD score.

Unadjusted mortality rates were computed as the ratio of risk categoryspecific deaths per 1000 patient years of follow-up. Cox regression models were used to compare mortality for waiting list candidates and transplant recipients, at equal duration since wait-listing, adjusted for age, sex, race, diagnosis, education, insurance coverage and updated (time-dependent) MELD score and $\triangle M E L D$ (17). We computed $\triangle M E L D$, a measure of how rapidly the candidate's MELD is changing, as the change in MELD score divided by the length of the time interval between reported dates of change.

The covariate-adjusted hazard ratio for mortality risk of transplant compared to mortality risk on the waiting list was estimated for the study population as a whole. Separate models were also fitted for several MELD subgroups. Lastly, non-proportional (piecewise proportional hazards) Cox models were used to estimate mortality hazard ratios for post-transplant follow-up time windows of $0-7,8-30$ and $31-365$ days compared to corresponding waiting list candidates.

\section{Results}

Characteristics of the study population at the time of placement on the waiting list are shown in Table 1. Just over $60 \%$ of candidates were aged 50 or older at listing. Males made up $64 \%$ of the cohort and over $87 \%$ of the candidates were white. Hispanic ethnicity was noted for $14.6 \%$ of candidates. The most common diagnosis group was noncholestatic cirrhosis; hepatitis $\mathrm{C}$ cirrhosis was the most common individual diagnosis (32\%).

At the time of listing, more than one-half of candidates had a MELD score less than 15 and $9 \%$ had a score above 26 (Figure 1). The distribution of MELD scores at the time of transplant showed a shift toward higher scores (more than $25 \%$ above 26), although $24 \%$ of transplants were performed at MELD scores less than 15 (Figure 2). The proportion of transplant recipients with a MELD score at transplant less than 12 was $10 \%$.

A total of 1538 pre-transplant deaths and 255 posttransplant deaths were observed (Table 2). The unadjusted 
Table 1: Characteristics of study population at wait-listing ( $\mathrm{n}=$ 12 996)

\begin{tabular}{|c|c|c|}
\hline & $\mathrm{N}$ & $\%$ \\
\hline \multicolumn{3}{|l|}{ Age } \\
\hline 18-39 & 1161 & 8.9 \\
\hline $40-49$ & 4001 & 30.8 \\
\hline $50-59$ & 5307 & 40.8 \\
\hline$\geq 60$ & 2527 & 19.4 \\
\hline \multicolumn{3}{|l|}{ Sex } \\
\hline Male & 8291 & 63.8 \\
\hline Female & 4705 & 36.2 \\
\hline \multicolumn{3}{|l|}{ Race } \\
\hline Asian & 468 & 3.6 \\
\hline African American & 963 & 7.4 \\
\hline Caucasian & 11246 & 86.5 \\
\hline Other & 319 & 2.5 \\
\hline \multicolumn{3}{|l|}{ Ethnicity } \\
\hline Hispanic & 1900 & 14.6 \\
\hline Non-Hispanic & 11096 & 85.4 \\
\hline \multicolumn{3}{|l|}{ Diagnosis } \\
\hline Hepatitis C cirrhosis & 4189 & 32.2 \\
\hline Alcoholic cirrhosis & 2044 & 15.7 \\
\hline Hepatitis B cirrhosis & 534 & 4.1 \\
\hline Non-cholestatic cirrhosis (other) & 2644 & 20.3 \\
\hline Primary sclerosing cholangitis & 586 & 4.5 \\
\hline Primary biliary cirrhosis & 539 & 4.2 \\
\hline Cholestatic cirrhosis (other) & 30 & 0.2 \\
\hline Others & 2430 & 18.7 \\
\hline \multicolumn{3}{|l|}{ Education } \\
\hline High school or less & 4914 & 37.8 \\
\hline College & 3982 & 30.6 \\
\hline Missing & 4100 & 31.6 \\
\hline \multicolumn{3}{|l|}{ Insurance } \\
\hline Medicare/Medicaid & 3599 & 27.7 \\
\hline Private/self & 4073 & 31.3 \\
\hline Missing/other & 5324 & 41.0 \\
\hline
\end{tabular}

waiting list mortality rate was 217 deaths per 1000 patient years, compared to 184 deaths per 1000 patient years among transplant recipients. There was approximately 300-fold range of waiting list mortality rates between patients with MELD scores 6-11 and those with MELD scores of 40 and higher. Patients with MELD scores 40 and higher had unadjusted post-transplant mortality rates that were more than $50 \%$ greater than that of the MELD 6-11 category.

Overall covariate-adjusted mortality risk from the Cox regression model was $79 \%$ lower for liver transplant recipients compared to candidates on the waiting list (hazard ratio $=0.21 ; 95 \%$ confidence interval $=0.18-0.24 ; p<$ 0.001 ), indicating a significant survival benefit of liver transplantation. However, the salutary effect of transplantation on survival varied across the range of MELD scores (Figure 3). With 1 year of available post-transplant follow-up, MELD category 15-17 represented a transition point. Posttransplant mortality risk was more than three times higher than waiting list mortality for MELD scores 6-11 (hazard ratio $=3.64 ;$ 95\% confidence interval $=2.23-5.95 ; \mathrm{p}<$ $0.001)$. The hazard ratio was also greater than 1.0 among patients with MELD 12-14 (hazard ratio = 2.35; 95\% confidence interval 1.48-3.76; $p<0.001)$. Conversely, significant transplant survival benefit was observed at MELD scores 18 and higher, and the magnitude of transplant benefit increased with increasing MELD score (Figure 3).

The majority of patients with low MELD scores showed little to no progression over time. Among 1861 patients who were initially listed in the MELD (6-11) category and had at least 1 year of follow-up, 75.9\% remained in that category 1 year later. Less than $5 \%$ progressed into a MELD score category associated with significant survival benefit.

Some patients were assigned a MELD score of 40 by the OPTN even though their calculated score was higher, because current allocation policy caps the score. Among 570 patients with a capped MELD score, 469 (82\%) had an actual calculated MELD greater than 40 (range: 41-68). A separate Cox regression model for patients with MELD scores greater than or equal to 40 was fitted using the actual calculated MELD scores showed that mortality risk (and thus transplant benefit) for transplant recipients among these most ill patients remained stable (hazard ratio $=1.00 ; 95 \%$ confidence interval 0.89-1.11).

The observed contrast between post-transplant and waiting list mortality varied strongly across the follow-up period

Figure 1: Distribution of MELD scores at initial placement on waiting list.

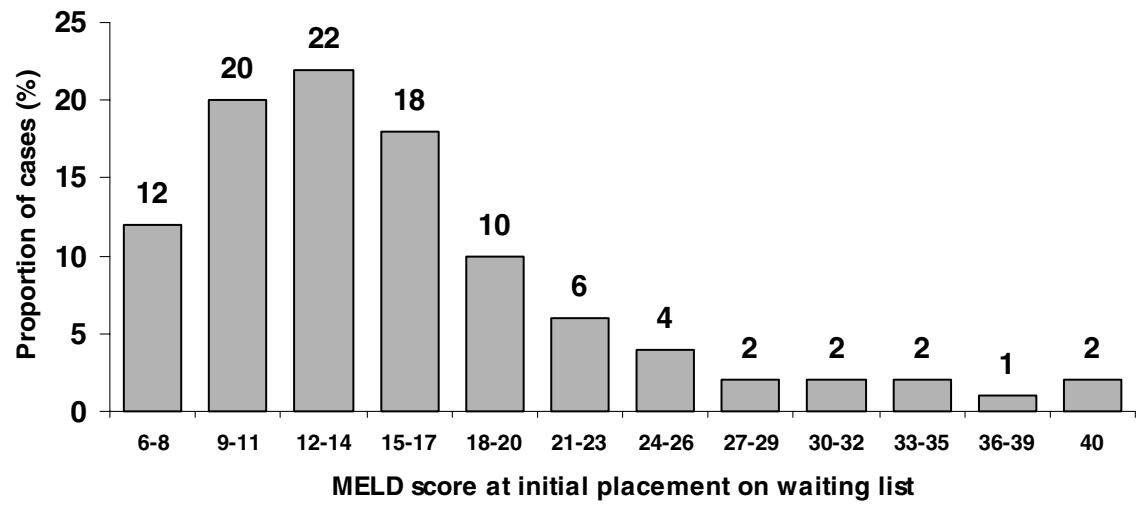



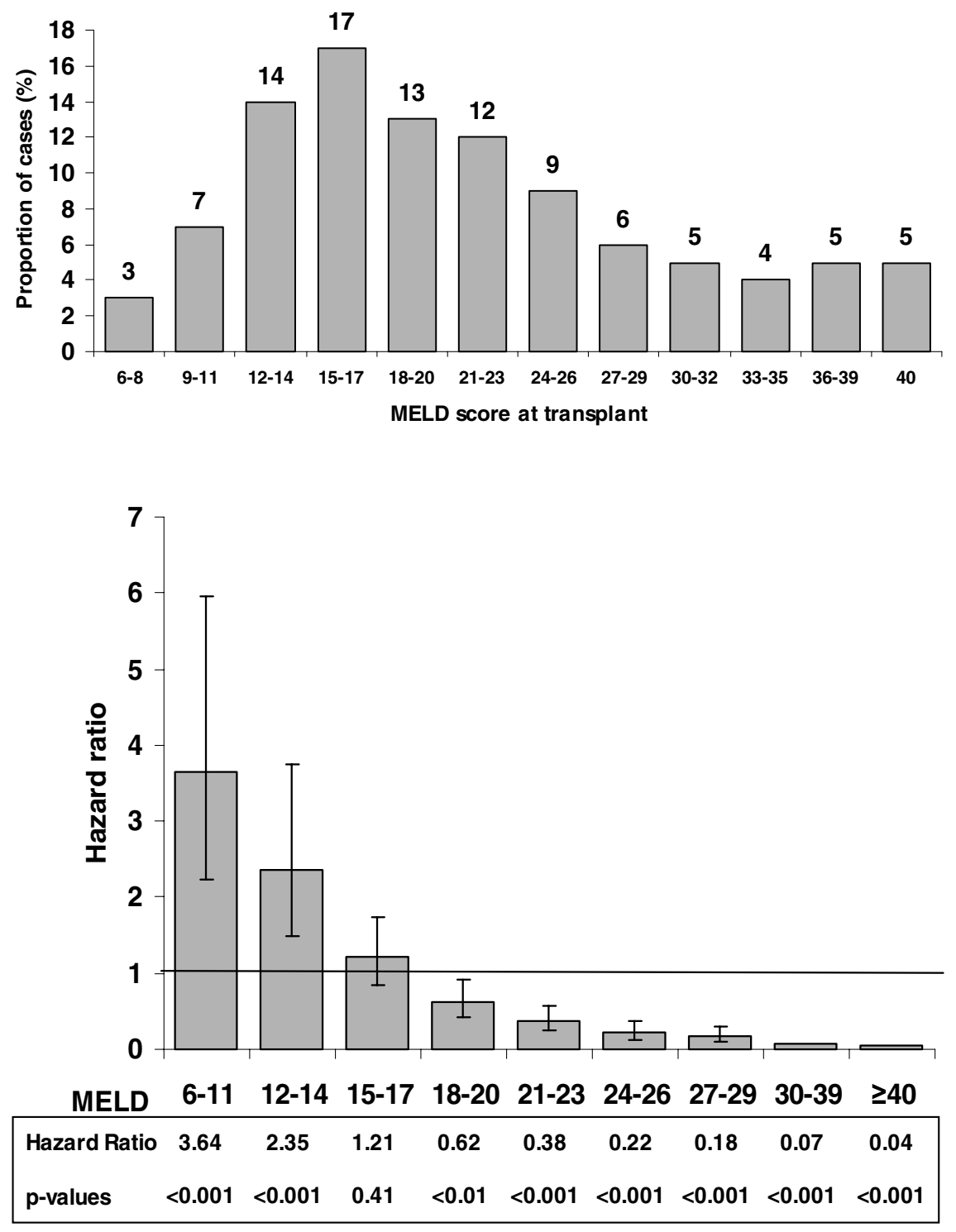

Figure 2: Distribution of MELD scores at transplant.

Figure 3: Comparison of mortality risk expressed as hazard ratio by MELD score for recipients of liver transplants compared to candidates on the liver transplant waiting list.

Table 2: Unadjusted waiting list and transplant mortality rates by MELD category

\begin{tabular}{|c|c|c|c|c|c|c|}
\hline \multirow[b]{2}{*}{ MELD } & \multicolumn{3}{|c|}{ Waiting list } & \multicolumn{3}{|c|}{ Transplant (1 year follow-up) } \\
\hline & Deaths & Patient years (PY) & Rate per 1000 PY & Deaths & Patient years (PY) & Rate per $1000 \mathrm{PY}$ \\
\hline $6-11$ & 130 & 2901 & 44.8 & 19 & 116 & 163.3 \\
\hline $12-14$ & 96 & 1830 & 52.5 & 23 & 180 & 127.4 \\
\hline $15-17$ & 181 & 1237 & 146.4 & 38 & 231 & 164.7 \\
\hline $18-20$ & 150 & 552 & 271.9 & 33 & 190 & 174.1 \\
\hline $21-23$ & 142 & 276 & 514.9 & 31 & 174 & 178.4 \\
\hline $24-26$ & 105 & 125 & 840.7 & 21 & 119 & 176.9 \\
\hline $27-29$ & 99 & 60 & 1663.8 & 18 & 92 & 195.9 \\
\hline $30-39$ & 348 & 75 & 4634.1 & 54 & 220 & 245.5 \\
\hline $40^{*}$ & 287 & 22 & 13152.7 & 18 & 68 & 264.6 \\
\hline Total & 538 & 7078 & 217.3 & 255 & 1390 & 183.5 \\
\hline
\end{tabular}

*Includes patients whose MELD score was capped at 40. 
Liver Transplant Survival Benefit

Table 3: Covariate-adjusted mortality hazard ratios (transplant: waiting list) by MELD score category and post-transplant follow-up time

\begin{tabular}{|c|c|c|c|c|c|c|c|c|c|}
\hline \multirow[b]{3}{*}{ MELD Category } & \multicolumn{9}{|c|}{ Days post-transplant } \\
\hline & \multicolumn{3}{|c|}{$0-7$ days } & \multicolumn{3}{|c|}{ 8-30 days } & \multicolumn{3}{|c|}{ 31-365 days } \\
\hline & $\mathrm{HR}^{*}$ & $(95 \% \mathrm{Cl}) \dagger$ & p-value & $\mathrm{HR}^{*}$ & $(95 \% \mathrm{Cl}) \dagger$ & p-value & $\mathrm{HR}^{*}$ & $(95 \% \mathrm{Cl}) \dagger$ & $\mathrm{p}$-value \\
\hline $6-11$ & 33.03 & $(13.74,79.22)$ & $<0.001$ & 9.26 & $(3.34,25.69)$ & $<0.001$ & 1.79 & $(0.89,3.59)$ & 0.10 \\
\hline $12-14$ & 27.91 & $(11.81,66.00)$ & $<0.001$ & 1.24 & $(0.17,8.96)$ & 0.83 & 1.76 & $(1.01,3.06)$ & 0.04 \\
\hline $15-17$ & 5.86 & $(2.57,13.35)$ & $<0.001$ & 2.52 & $(1.23,5.16)$ & 0.01 & 0.82 & $(0.52,1.28)$ & 0.37 \\
\hline $18-20$ & 2.83 & $(1.04,7.72)$ & 0.04 & 1.13 & $(0.46,2.78)$ & 0.79 & 0.48 & $(0.31,0.76)$ & 0.002 \\
\hline $21-29$ & 1.65 & $(1.00,2.73)$ & 0.05 & 0.50 & $(0.29,0.86)$ & 0.01 & 0.15 & $(0.11,0.22)$ & $<0.001$ \\
\hline 30-39 & 0.20 & $(0.09,0.43)$ & $<0.001$ & 0.09 & $(0.05,0.17)$ & $<0.001$ & 0.06 & $(0.04,0.08)$ & $<0.001$ \\
\hline $40 \ddagger$ & 0.11 & $(0.04,0.26)$ & $<0.001$ & 0.04 & $(0.01,0.10)$ & $<0.001$ & 0.02 & $(0.01,0.03)$ & $<0.001$ \\
\hline
\end{tabular}

*Hazard ratio for post-transplant mortality risk based on MELD score at transplant compared to wait-listing while at the same MELD score.

†95\% confidence interval.

$\ddagger$ Includes patients whose MELD score was capped at 40 .

(Table 3). Generally, post-transplant mortality within each MELD category decreased as follow-up time increased. At MELD 6-11, post-transplant mortality was significantly higher than waiting list mortality for the first post-transplant month, while recipients transplanted at MELD scores of 30 and higher had a significant survival benefit within the first post-transplant week. Recipients with MELD scores 2129 demonstrated significant survival benefit after the first post-transplant week.

\section{Discussion}

The ability of MELD to predict mortality in patients with chronic liver disease has contributed to the evolution of liver allocation policy in the United States $(9,10)$. It is established that MELD is an important predictor of waiting list mortality $(11,17,18)$, and several authors have recently reported that MELD is predictive of post-transplant mortality risk $(6,19)$. One study failed to show a significant relationship between pre-transplant MELD score and posttransplant outcome, but this report was from a singlecenter study with limited sample size (20). In our study, we found approximately a 300-fold range of mortality risk across the MELD spectrum for patients on the waiting list, and a 1.5-fold range for post-transplant mortality risk. The availability of serial MELD data allows detailed analyses of the survival benefit of liver transplantation to be performed, comparing waiting list and post-transplant mortality risk within MELD categories.

This study demonstrates significant overall liver transplant survival benefit for patients with chronic liver disease, and provides important validation of the procedure as lifesaving therapy. Deceased donor liver transplant recipients have an overall covariate-adjusted mortality risk that is less than one-quarter that of patients on the waiting list; this finding holds with and without the inclusion of $\triangle M E L D$ as a covariate.

Liver transplant survival benefit is not evenly distributed across the range of MELD scores. This is particularly use- ful in considerations of organ allocation, where relative survival benefit among a group of candidates must be determined. On the other hand, the physician and candidate faced with a decision regarding wait-listing or acceptance of an offer of a donor liver must consider whether that particular candidate's expected lifetime with a transplant, given their current medical condition as reflected by their MELD score or other factors, is predicted to exceed that expected if the transplant is not performed. Our analyses facilitate discussion of both these issues.

At MELD scores 18 and higher, significant and progressively increasing survival benefit was demonstrated. At the highest end of the risk spectrum, there does not appear to be a MELD score above which transplantation is clearly futile. In a supplementary analysis, a Cox model fitted to the subgroup of patients with uncapped MELD scores of 40 and greater showed that the mortality hazard ratio continued to favor transplant. However, the rate of transfer to inactive status on the waiting list increases at higher MELD scores (data not shown), suggesting that effective clinical decision making and recipient selection is at work among these sick candidates. In fact, at the highest end of the MELD scale, waiting list mortality risk is extremely high, whereas post-transplant mortality risk rises much more gradually. These relationships help to explain the progressively higher benefit of liver transplantation as MELD increases.

In contrast to the findings for patients with high MELD scores, post-transplant mortality risk for the nearly one in four recipients who received liver transplants for chronic liver disease at a MELD score less than 15 was significantly higher than for comparable candidates on the waiting list. This suggests that liver transplant is more hazardous than remaining on the waiting list in such cases, based on 1 year of post-transplant follow-up. With longer follow-up, a survival benefit for low MELD patients may eventually be demonstrated, but in relative terms that benefit is likely to be of lesser magnitude than for those with higher MELD scores. Moreover, three-quarters of patients with MELD 
scores between 6 and 11 at listing continue to have MELD scores in that range 1 year later, suggesting that the risk of rapid progression is low.

On initial examination, it may be surprising that so many patients with low MELD scores receive liver transplants, as the current allocation policy is intended to direct donor livers to high MELD score candidates. Two related factors explain this finding. First, the MELD scores of the highest ranking candidate in each OPO vary considerably, reflecting the marked heterogeneity in the acuity of liver transplant candidates across the United States (21). Second, organs are offered to chronic liver disease candidates in descending MELD score order within the OPO service area where the donor is identified before they are offered to candidates outside that OPO. Thus, one-half of all livers are offered first to candidates with MELD scores less than 18 (data not shown). These factors collectively lead to the current situation in which over $40 \%$ of all liver transplants are given to patients with MELD scores below 18.

This study has implications for liver allocation policy in the United States. The current ordering of liver transplant candidates with chronic liver disease appears to be suboptimal. Distribution of donor livers to candidates with higher MELD scores in a larger initial geographic unit than the OPO, extending to the regional level, would simultaneously steer a higher proportion of scarce organs to patients likely to benefit from them and reduce the probability of transplant for those with less likelihood of benefit. The latter element is important, given that candidates with very low MELD scores cannot yet be shown to benefit from a transplant, and may even be harmed by one. The Board of Directors of the OPTN recently approved such a modification to the national allocation system for deceased donor livers in the United States (22).

Separately, establishment of a minimum MELD score for placement on the waiting list would be a more direct method of reducing the proportion of transplants given to patients with low MELD scores. Selection of a minimum listing MELD score needs to be informed by several considerations. With sufficiently long follow-up, a subset of patients with MELD scores below 18 may ultimately be shown to derive significant benefit. It may be imprudent, therefore, to suggest a minimum MELD score for listing that is too close to the currently calculated transplant benefit transition range of 15-17. On the downside, if patients were no longer able to be listed for transplant at low MELD scores, it might be more difficult for transplant physicians to study the course of end-stage liver disease. It has been suggested that transplant teams might not be permitted by third-party payers to follow patients referred for transplantation unless they are placed on the waiting list. These considerations must be weighed against the potential harm of doing a liver transplant that does not significantly prolong the life of the recipient, particularly as candidates with higher MELD scores and greater expected benefit are avail- able on the waiting list. Reevaluation of individual benefit calculations will be useful when longer follow-up becomes available, although it will not likely change the ordering of benefit by MELD.

In summary, our analysis adds important data to the continuing evolution in organ allocation policy. These results suggest that transplants are being performed for some candidates who have a higher risk of dying from the transplant procedure than they have of dying from their underlying liver disease. Rather than relying solely on the risk of pre-transplant death, the survival benefit among candidates should be considered as a component of allocation policy in order to direct organs to those most likely to benefit from the procedure. The current practice of transplanting substantial number of patients at low MELD scores, with associated lack of demonstrable transplant benefit, should be carefully reconsidered.

\section{Acknowledgment}

This study was funded by the Scientific Registry of Transplant Recipients and supported by contract number 231-00-0116 from the Health Resources and Services Administration, U.S. Department of Health and Human Services. The views expressed in this papers are those of the authors and are not necessarily those of the US Government.

\section{References}

1. Christensen E, Gunson B, Neuberger J. Optimal timing of liver transplantation for patients with primary biliary cirrhosis: use of prognostic modelling. J Hepatol 1999; 30: 285-292.

2. Ricci P, Therneau TM, Malinchoc M et al. A prognostic model for the outcome of liver transplantation in patients with cholestatic liver disease. Hepatology 1997; 25: 672-677.

3. Grambsch PM, Dickson ER, Wiesner RH, Langworthy A. Application of the Mayo primary biliary cirrhosis survival model to Mayo liver transplant patients. Mayo Clin Proc 1989; 64: 699-704.

4. 2002 Annual Report of the U.S. Organ Procurement and Transplantation Network and the Scientific Registry for Transplant Recipients: Transplant Data 1992-2001: Department of Health and Human Services, Health Resources and Services Administration, Office of Special Programs, Division of Transplantation, Rockville, MD; United Network for Organ Sharing, Richmond, VA; University Renal Research and Education Association, Ann Arbor, Ml; 2002.

5. Delmonico FL, Jenkins RL, Freeman $\mathrm{R}$ et al. The high-risk liver allograft recipient. Should allocation policy consider outcome? Arch Surg 1992; 127: 579-584.

6. Onaca NN, Levy MF, Sanchez EQ et al. A correlation between the pretransplantation MELD score and mortality in the first two years after liver transplantation. Liver Transpl 2003; 9: 117-123.

7. Organ Procurement and Transplantation Network. Policy 3. Organ Distribution. http://www.optn.org/policiesAndBylaws/policies. asp. Accessed 04/18/2003. (Accessed at http://www.optn.org/ policiesAndBylaws/policies.asp.)

8. Wolfe RA, Ashby VB, Milford EL et al. Comparison of mortality in all patients on dialysis, patients on dialysis awaiting transplantation, and recipients of a first cadaveric transplant. N Engl J Med 1999; 341: 1725-1730. 


\section{Liver Transplant Survival Benefit}

9. Malinchoc M, Kamath PS, Gordon FD, Peine CJ, Rank J, ter Borg PC. A model to predict poor survival in patients undergoing transjugular intrahepatic portosystemic shunts. Hepatology 2000 31: 864-871.

10. Kamath PS, Wiesner $\mathrm{RH}$, Malinchoc M et al. A model to predict survival in patients with end-stage liver disease. Hepatology 2001 33: 464-470.

11. Wiesner RH, McDiarmid SV, Kamath PS et al. MELD and PELD: application of survival models to liver allocation. Liver Transplantation 2001; 7: 567-580.

12. McDiarmid SV, Anand R, Lindblad AS. The Principal Investigators and Institutions of the Studies of Pediatric Liver Transplantation Research Group. Development of a pediatric end-stage liver disease score to predict poor outcome in children awaiting liver transplantation. Transplantation 2002; 74: 173-181.

13. Social Security Administration Death Master File. Federal Computer Products Center, National Technical Information Service U.S. Department of Commerce, Springfield, VA.

14. Dickinson DM, Ellison MD, Webb RL. Data sources and structure. Am J Transplant 2003; 3(Suppl. 4): 13-28.

15. Wolfe RA, Webb RL, Dickinson DM et al. Analytical approaches for transplant research. Am J Transplant 2003; 3(Suppl. 4): 10313.
16. Organ Procurement and Transplantation Network. Policy 3.6. Allocation of deceased livers. Available at http://www.optn.org/ PoliciesandBylaws/policies/pdfs/policy_8.pdf (Accessed 02/02/ 2004).

17. Merion RM, Wolfe RA, Dykstra DM, Leichtman AB, Gillespie $B$, Held PJ. Longitudinal assessment of mortality risk among candidates for liver transplantation. Liver Transpl 2003; 9: 1218.

18. Wiesner R, Edwards E, Freeman R et al. Model for end-stage liver disease (MELD) and allocation of donor livers. Gastroenterology 2003; 124: 91-96.

19. Saab S, Wang $V$, Ibrahim AB et al. MELD score predicts 1-year patient survival post-orthotopic liver transplantation. Liver Transpl 2003; 9: 473-476.

20. Akyildiz M, Karasu Z, Arikan C et al. Impact of pretransplant MELD score on posttransplant outcome in living donor liver transplantation. Transplant Proc 2004; 36: 1442-1444.

21. Freeman RB, Wiesner $\mathrm{RH}$, Edwards $\mathrm{E}$, Harper $\mathrm{A}$, Merion $\mathrm{R}$, Wolfe $R$. Results of the first year of the new liver allocation plan. Liver Transpl 2004; 10: 7-15.

22. http://www.optn.org/PoliciesandBylaws/policies/pdfs/policy_8.pdf Accessed 08/20/2004. 